\title{
BILIPSCHITZ EQUIVALENCE OF POLYNOMIALS
}

\author{
ARNAUD BODIN
}

\begin{abstract}
We study a family of two variables polynomials having moduli up to bilipschitz equivalence: two distinct polynomials of this family are not bilipschitz equivalent. However any level curve of the first polynomial is bilipschitz equivalent to a level curve of the second.
\end{abstract}

\section{Global Bilipschitz equivalence}

Let $\mathbb{K}$ be $\mathbb{R}$ or $\mathbb{C}$. For polynomial maps $f, g: \mathbb{K}^{n} \rightarrow \mathbb{K}$ we introduce two notions of bilipschitz equivalence: a level equivalence (a hypersurface $(f=c)$ is sent to a hypersurface $\left.\left(g=c^{\prime}\right)\right)$ and a global equivalence (any level $(f=c)$ is sent to another level $\left.\left(g=c^{\prime}\right)\right)$.

- $\mathbb{K}^{n}$ is endowed with the Euclidean canonical metric.

- A map $\Phi: \mathbb{K}^{n} \rightarrow \mathbb{K}^{n}$ is Lipschitz if there exists $K>0$ such that for all $x, y \in \mathbb{K}^{n}$ :

$$
\|\Phi(x)-\Phi(y)\| \leqslant K\|x-y\| .
$$

- A map $\Phi: \mathbb{K}^{n} \rightarrow \mathbb{K}^{n}$ is bilipschitz if it is a homeomorphism, Lipschitz and $\Phi^{-1}$ is also Lipschitz. Equivalently, $\Phi$ is bijective and there exists $K>0$ such that $\frac{1}{K}\|x-y\| \leqslant\|\Phi(x)-\Phi(y)\| \leqslant K\|x-y\|$.

- Two sets $\mathcal{C}$ and $\mathcal{C}^{\prime}$ of $\mathbb{K}^{2}$ are bilipschitz equivalent if there exists a bilipschitz map $\Phi: \mathbb{K}^{n} \rightarrow \mathbb{K}^{n}$ such that $\Phi(\mathcal{C})=\mathcal{C}^{\prime}$.

- Two functions $f, g: \mathbb{K}^{n} \rightarrow \mathbb{K}$ are right-bilipschitz equivalent if there exists a bilipschitz map $\Phi: \mathbb{K}^{n} \rightarrow \mathbb{K}^{n}$ such that $g \circ \Phi=f$.

- Two functions $f, g: \mathbb{K}^{n} \rightarrow \mathbb{K}$ are left-right-bilipschitz equivalent if there exist a bilipschitz map $\Phi: \mathbb{K}^{n} \rightarrow \mathbb{K}^{n}$ and a bilipschitz map $\Psi: \mathbb{K} \rightarrow \mathbb{K}$ such that $g \circ \Phi=$ $\Psi \circ f$.
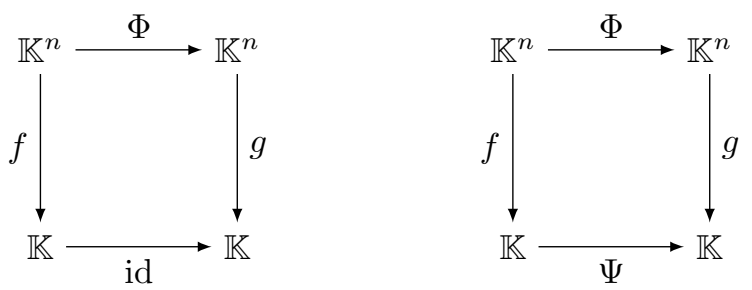

Figure 1. Two commutative diagrams. On the left: right-bilipschitz equivalence. On the right: left-right-bilipschitz equivalence.

Remarks:

Date: February 25, 2020.

2010 Mathematics Subject Classification. Primary 58K60 ; Sec. 12E05.

Key words and phrases. Bilipschitz geometry, Polynomials, Moduli.

Acknowledgment. This work was supported by the ANR project "LISA" (ANR-17-CE40-0023-01). 
- A map $\Phi: \mathbb{K}^{n} \rightarrow \mathbb{K}^{n}$ can be $C^{1}$ but not Lipschitz. Hence (bi-)Lipschitz is not an intermediate case between smooth and continuous. This is due to the noncompactness: for instance $\Phi: \mathbb{R} \rightarrow \mathbb{R}, x \mapsto x^{2}$ is $C^{1}$ but not Lipschitz.

- For similar reasons an algebraic automorphism of $\mathbb{K}^{n}$ does not necessarily provide a bilipschitz equivalence. For instance $f(x, y)=y$ and $g=y+x^{2}$ are algebraically equivalent using the map $\Phi:(x, y) \mapsto\left(x, y-x^{2}\right)$, but $\Phi$ is not bilipschitz.

It is clear that bilipschitz equivalence implies topological equivalence (i.e. when $\Phi$ and $\Psi$ are only homeomorphisms). The main question is: does topological equivalence implies bilipschitz equivalence? The answer is negative.

We will actually prove more. A theorem of Fukuda asserts that in a family of polynomials there is only a finite number of different types, up to topological equivalence, see [4], [3]. However the following theorem proves that the family of polynomials $f_{s}(x, y)=$ $x\left(x^{2} y^{2}-s x y-1\right)$ has moduli for bilipschitz equivalence, i.e. any two polynomials in this family are not right-bilipschitz equivalent.

Theorem 1. Consider the family of polynomial in $\mathbb{K}[x, y]$ :

$$
f_{s}(x, y)=x\left(x^{2} y^{2}-s x y-1\right) .
$$

- $\mathbb{K}=\mathbb{R}$. Any two polynomials $f_{s}$ and $f_{s^{\prime}}$ with $s, s^{\prime} \in \mathbb{R}, s \neq s^{\prime}$ are not rightbilipschitz equivalent. However the special levels $\left(f_{0}=0\right)$ and $\left(f_{1}=0\right)$ are bilipschitz equivalent and the generic levels $\left(f_{0}=1\right)$ and $\left(f_{1}=1\right)$ are bilipschitz equivalent.

$-\mathbb{K}=\mathbb{C}$. Fix $s \in \mathbb{C}$, with $s^{2}+3 \neq 0$. For all but finitely many $s^{\prime} \in \mathbb{C}, f_{s}$ and $f_{s^{\prime}}$ are not right-bilipschitz equivalent. However, if $s^{2}+4 \neq 0$ and $s^{2}+4 \neq 0$, the polynomials $f_{s}$ and $f_{s^{\prime}}$ are topologically equivalent.

This is a version at infinity of a result by Henry and Parusiński, [5]. Our polynomials $f_{s}$ have only one special level $\left(f_{s}=0\right)$ which plays the role of the singular level of the local examples of [5]. We recall that for a polynomial map $f: \mathbb{K}^{n} \rightarrow \mathbb{K}$ there is a notion of generic levels $(f=c)$ and a finite number of special levels whose topology is not the generic one. Special levels can be due to the presence of a singular point or to singularity at infinity as this the case in our examples. We will in fact prove a non bilipschitz equivalence "at infinity", after defining that two functions are bilipschitz equivalent at infinity if they are bilipschitz equivalent outside some compact sets.

Acknowledgments. I thank Vincent Grandjean, Anne Pichon and Patrick PopescuPampu for their encouragements and the referees for their comments.

\section{LEVELS ARE BILIPSCHITZ EQUIVALENT}

Lemmas 2 and 3 in this section will prove the bilipschitz real equivalence of theorem 1. Let

$$
f_{s}(x, y)=x\left(x^{2} y^{2}-s x y-1\right)
$$

which, in this section, is considered as a family of polynomials in $\mathbb{R}[x, y]$.

Lemma 2. The levels $\left(f_{0}=0\right)$ and $\left(f_{1}=0\right)$ are bilipschitz equivalent, that is to say there exists a bilipschitz map $\Phi: \mathbb{R}^{2} \rightarrow \mathbb{R}^{2}$ such that $\Phi\left(\left(f_{0}=0\right)\right)=\left(f_{1}=0\right)$.

In other words, the (unique) special fibers of $f_{0}$ and $f_{1}$ are bilipschitz equivalent. 
Proof.

\section{Definition of $\Phi$.}

- Let $\sigma=\frac{\sqrt{5}+1}{2}$ be the positive root of $z^{2}-z-1=0$. Let $\tau=\frac{\sqrt{5}-1}{2}$ be the positive root of $z^{2}+z-1=0$.

- We define a map $\Phi: \mathbb{R}^{2} \rightarrow \mathbb{R}^{2}$ by the following formulas:

- For $(x, y) \in(x y=1)$ we define:

$$
\Phi(x, y)=(a x, b y) \quad \text { with } a b=\sigma,
$$

such that $(a, b)$ depends on $(x, y)$ in the following way:

$$
\begin{cases}(a, b)=(\sigma, 1) & \text { if }|x| \leqslant \frac{1}{2} \\ (a, b)=(1, \sigma) & \text { if }|x| \geqslant 2\end{cases}
$$

and extended to a smooth map for $\frac{1}{2} \leqslant|x| \leqslant 2$ so that the relation $a b=\sigma$ is always satisfied on $(x y=1)$.

- For $(x, y) \in(x y=-1)$ we similarly define $\Phi(x, y)=(a x, b y)$ with $a b=\tau$, and $(a, b)=(\tau, 1)$ for $|x| \leqslant \frac{1}{2},(a, b)=(1, \tau)$ for $|x| \geqslant 2$ and extended in a smooth map for $\frac{1}{2} \leqslant|x| \leqslant 2$.

$-\Phi(0, y)=(0, y)$ for all $y \in \mathbb{R}$.

- $\Phi(x, y)=(x, y)$ for $(x, y)$ outside a neighborhood $\mathcal{N}$ of radius 1 of $(x y=$ 1) $\cup(x y=-1)$.

- $\Phi$ is extended on $\mathcal{N}$ to a bilipschitz homeomorphism $\Phi: \mathbb{R}^{2} \rightarrow \mathbb{R}^{2}$.
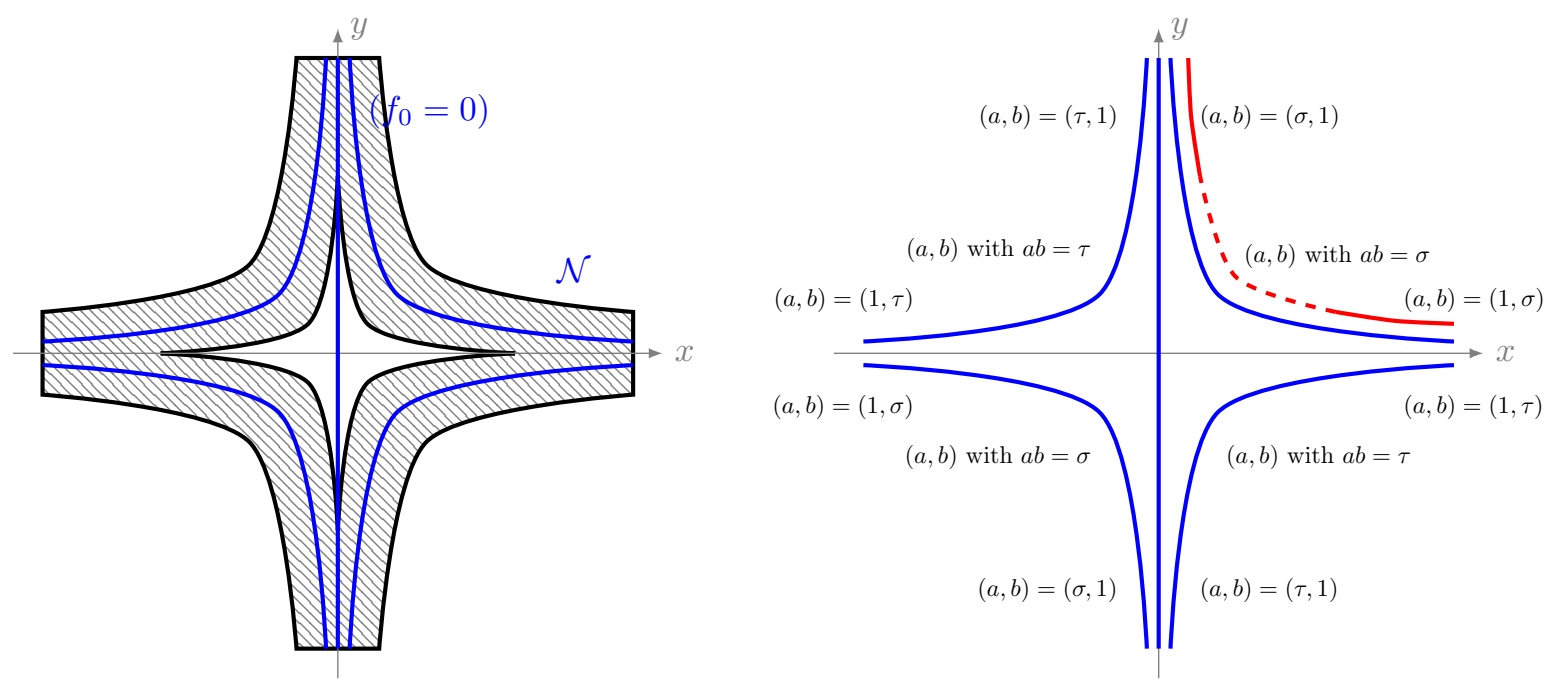

Figure 2. The definition of $\Phi$. Left: the level, a neighborhood of the level.

Right: the values $(a, b)$ for the definition of $\Phi(x, y)=(a x, b y)$ on the level.

- The only point to prove is that the formulas actually yield a bilipschitz map around the axis. For instance let $\left(x_{1}, y_{1}\right) \in(x y=1)$ with $x_{1}>2$, so that $\Phi\left(x_{1}, y_{1}\right)=$ $\left(x_{1}, \sigma y_{1}\right)$ and $\left(x_{2}, y_{2}\right) \in(x y=-1)$ with $x_{2}>2$ and $\Phi\left(x_{2}, y_{2}\right)=\left(x_{2}, \tau y_{2}\right)$. Then

$$
\begin{aligned}
\left\|\Phi\left(x_{1}, y_{1}\right)-\Phi\left(x_{2}, y_{2}\right)\right\| & =\left\|\left(x_{1}-x_{2}, \sigma y_{1}-\tau y_{2}\right)\right\| \\
& \leqslant \|\left(x_{1}-x_{2}, 2 \sigma\left(y_{1}-y_{2}\right) \|\right. \\
& \leqslant 2 \sigma\left\|\left(x_{1}-x_{2}, y_{1}-y_{2}\right)\right\|
\end{aligned}
$$

(using that $y_{1}-y_{2}=\left|y_{1}\right|+\left|y_{2}\right|$ ). A similar bound holds for $\Phi^{-1}$ on this branch.

Then $\Phi: \mathbb{R}^{2} \rightarrow \mathbb{R}^{2}$ is a bilipschitz homeomorphism. 


\section{Equivalence.}

- Let $f(x, y)=f_{0}(x, y)=x\left(x^{2} y^{2}-1\right)$ and $g(x, y)=f_{1}(x, y)=x\left(x^{2} y^{2}-x y-1\right)$.

- By definition of $\Phi, \Phi(0, y)=(0, y)$ so that the component $(x=0) \subset(f=0)$ is sent by $\Phi$ to $(x=0) \subset(g=0)$.

- Let $(x, y) \in(x y=1) \subset\left(x^{2} y^{2}=1\right) \subset(f=0)$. For such $(x, y), \Phi(x, y)=(a x, b y)$ with $a b=\sigma$.

- Let $\tilde{g}(x, y)=x^{2} y^{2}-x y-1$ :

$$
\tilde{g} \circ \Phi(x, y)=\tilde{g}(a x, b y)=a^{2} b^{2} x^{2} y^{2}-a b x y-1=\sigma^{2}(x y)^{2}-\sigma x y-1 .
$$

As $x y=1$ we get:

$$
\tilde{g} \circ \Phi(x, y)=\sigma^{2}-\sigma-1=0,
$$

by definition of $\sigma$. Then $\Phi(x, y) \subset(\tilde{g}=0) \subset(g=0)$. A similar reasoning holds for $(x y=-1)$.

We now prove that two generic fibers are also bilipschitz equivalent.

Lemma 3. The levels $\left(f_{0}=1\right)$ and $\left(f_{1}=1\right)$ are bilipschitz equivalent, that is to say there exists a bilipschitz map $\Phi: \mathbb{R}^{2} \rightarrow \mathbb{R}^{2}$ such that $\Phi\left(\left(f_{0}=1\right)\right)=\left(f_{1}=1\right)$.

Proof.

- Parameterization of $\left(f_{0}=1\right)$. The curve $\left(f_{0}=1\right)$ has equation $x^{3} y^{2}-x-1=0$ and a parameterization $(x, y)$ is given by

$$
\left.\left.\left.y_{+}=\sqrt{\frac{1}{x^{2}}+\frac{1}{x^{3}}} \quad \text { or } \quad y_{-}=-\sqrt{\frac{1}{x^{2}}+\frac{1}{x^{3}}} \quad \text { for } x \in\right]-\infty,-1\right] \cup\right] 0,+\infty[\text {. }
$$
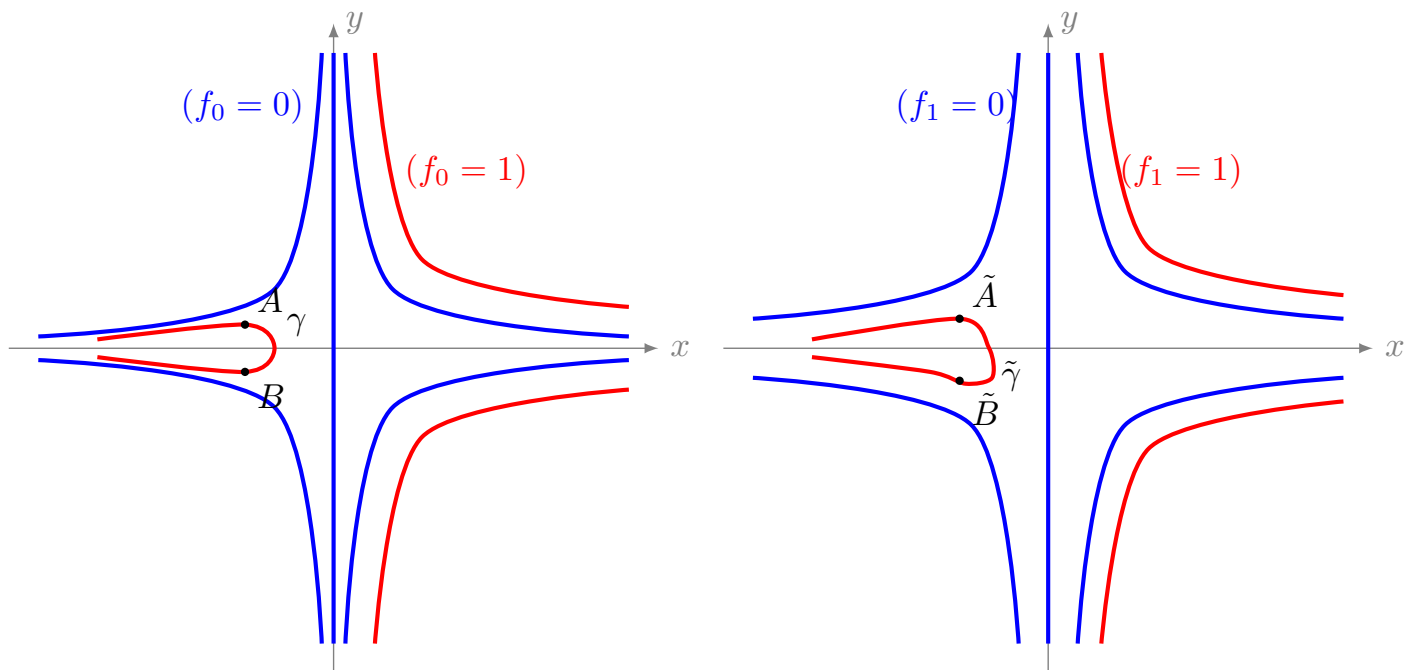

Figure 3 . The levels $\left(f_{0}=1\right)$ and $\left(f_{1}=1\right)$.

- Parameterization of $\left(f_{1}=1\right)$. The curve $\left(f_{1}=1\right)$ has equation $x^{3} y^{2}-x^{2} y-$ $x-1=0$, a parameterization is given by:

$Y_{+}=\frac{1}{2 x}+\frac{1}{2} \sqrt{\frac{5}{x^{2}}+\frac{4}{x^{3}}} \quad$ or $\quad Y_{-}=\frac{1}{2 x}-\frac{1}{2} \sqrt{\frac{5}{x^{2}}+\frac{4}{x^{3}}} \quad$ for $\left.\left.\left.x \in\right]-\infty,-\frac{5}{4}\right] \cup\right] 0,+\infty[$.

\section{- Definition of $\Phi$.}


- Case $x>0 . \Phi$ is defined on $\left(f_{0}=1\right)$ using the parameterization by the formula $\Phi(x, y)=\left(x, Y_{+}\right)$, for $(x, y) \in\left(f_{0}=1\right)$ with $x>0$ and $y>0$; $\Phi(x, y)=\left(x, Y_{-}\right)$, for $(x, y) \in\left(f_{0}=1\right)$ with $x>0$ and $y<0$.

- Case $x \leqslant-2$. $\Phi$ is defined by the same formulas $\Phi(x, y)=\left(x, Y_{+}\right)$(for $\left.y>0\right)$ or $\Phi(x, y)=\left(x, Y_{-}\right)($for $y<0)$.

- Case $-2 \leqslant x \leqslant-1$. (Note: we do not use the above formulas in the neighborhood of the point $(-1,0)$ because the map $y_{+} \mapsto Y_{+}$is not bilipschitz near this point.) Let $A, B$ be the two points of $(x=-2) \cap\left(f_{0}=1\right)$. Let $\tilde{A}, \tilde{B}$ be their images by $\Phi$ (i.e. $A, B$ belong $(x=-2) \cap\left(f_{1}=1\right)$ ). Let $\gamma$ be the compact part of $\left(f_{0}=1\right)$ between $A$ and $B$ and $\tilde{\gamma}$ be the compact part of $\left(f_{1}=1\right)$ between $\tilde{A}$ and $\tilde{B}$. We extend $\Phi$ in a bilipschitz way from $\gamma$ to $\tilde{\gamma}$. This is possible as $\gamma$ and $\tilde{\gamma}$ are two compact connected components of a smooth algebraic curve. $\Phi$ is now defined everywhere on $\left(f_{0}=1\right)$.

- We extend $\Phi$ on $\mathbb{R}^{2}$ to a bilipschitz map $\Phi: \mathbb{R}^{2} \rightarrow \mathbb{R}^{2}$. For instance we may suppose $\Phi$ is the identity outside a tubular neighborhood or radius 1 of $\left(f_{0}=1\right)$.

- Bilipschitz on $\left(f_{0}=1\right)$. It remains to justify that $\Phi$ is actually a bilipschitz map from $\left(f_{0}=1\right)$ to $\left(f_{1}=1\right)$.

- Case $x>0$ and $x \rightarrow 0$. Hence $y \rightarrow \pm \infty$. Then $y_{+} \sim \frac{1}{x^{3 / 2}}$ and $Y_{+} \sim \frac{1}{x^{3 / 2}} \sim y_{+}$ so that the map $\Phi\left(x, y_{+}\right)=\left(x, Y_{+}\right)$is bilipschitz. The same applies for $y_{-}$and $Y_{-}$.

- Case $x \rightarrow+\infty$. Hence $y \rightarrow 0$. Then $y_{+} \sim \frac{1}{x}$ and $Y_{+} \sim \frac{\sqrt{5}+1}{2} \cdot \frac{1}{x} \sim \sigma y_{+}$. Then, as in the proof of proposition $2, \Phi\left(x, y_{+}\right)=\left(x, Y_{+}\right)$is bilipschitz. The same applies for $y_{-}$and $Y_{-} \sim \tau y_{-}$with $\tau=\frac{\sqrt{5}-1}{2}$.

- Case $x \rightarrow-\infty$. It is similar to the previous case: $Y_{+} \sim \tau y_{+}, Y_{-} \sim \sigma y_{-}$.

\section{Moduli}

The following theorem proves that under bilipschitz equivalence at infinity a family of polynomials can have moduli. It is a version at infinity of the example of Henry and Parusiński [5]. Two functions $f, g: \mathbb{K}^{n} \rightarrow \mathbb{K}$ are right-bilipschitz equivalent at infinity if there exist compact sets $C, C^{\prime}$ and a bilipschitz map $\Phi: \mathbb{K}^{n} \backslash C \rightarrow \mathbb{K}^{n} \backslash C^{\prime}$ such that $g \circ \Phi=f$.

Using this notion, we will prove the moduli affirmation of theorem 1 with the following refinement.

\section{Theorem 1'.}

$$
f_{s}(x, y)=x\left(x^{2} y^{2}-s x y-1\right) \in \mathbb{K}[x, y] .
$$

$-\mathbb{K}=\mathbb{R}$. Any two polynomials $f_{s}$ and $f_{s^{\prime}}$ with $s, s^{\prime} \in \mathbb{R}, s \neq s^{\prime}$ are not rightbilipschitz equivalent at infinity (hence not globally right-bilipschitz equivalent). Moreover they are also not left-right-equivalent if we assume $\Phi$ analytic at infinity.

$-\mathbb{K}=\mathbb{C}$. Fix $s \in \mathbb{C}$, with $s^{2}+3 \neq 0$. For all but finitely many (explicit) $s^{\prime} \in \mathbb{C}$, $f_{s}$ and $f_{s^{\prime}}$ are not right-bilipschitz equivalent at infinity (hence not globally rightbilipschitz equivalent).

\subsection{Preliminaries.}

- Let $f_{s}(x, y)=x\left(x^{2} y^{2}-s x y-1\right)=x^{3} y^{2}-s x^{2} y-x$. 
- Then $\partial_{x} f_{s}(x, y)=3 x^{2} y^{2}-2 s x y-1$.

- The equation $3 z^{2}-2 s z-1=0$ has discriminant $\Delta=4\left(s^{2}+3\right)$ and two solutions:

$$
\alpha_{s}=\frac{s+\sqrt{s^{2}+3}}{3} \text { and } \beta_{s}=\frac{s-\sqrt{s^{2}+3}}{3} .
$$

- The polar curve $\Gamma_{s}:\left(\partial_{x} f_{s}=0\right)$, associated to the projection on the $y$-axis, has two components:

$$
\left(x y=\alpha_{s}\right) \quad \text { and } \quad\left(x y=\beta_{s}\right)
$$

parameterized by:

$$
\left(\alpha_{s} t, \frac{1}{t}\right) \text { and }\left(\beta_{s} t, \frac{1}{t}\right) \quad t \in \mathbb{K} \backslash\{0\} .
$$

- We compute the values of $f_{s}$ on the polar components. Near the point at infinity $(0: 1: 0)$, that is to say for $t \rightarrow 0$, we compute the values of $f_{s}$ on each branch of $\Gamma_{s}:$

$$
f_{s}\left(\alpha_{s} t, \frac{1}{t}\right)=\alpha_{s}\left(\alpha_{s}^{2}-s \alpha_{s}-1\right) t
$$

and

$$
f_{s}\left(\beta_{s} t, \frac{1}{t}\right)=\beta_{s}\left(\beta_{s}^{2}-s \beta_{s}-1\right) t .
$$

- We compare theses values for two branches at a same $y$-value:

$$
\frac{f_{s}\left(\alpha_{s} t, \frac{1}{t}\right)}{f_{s}\left(\beta_{s} t, \frac{1}{t}\right)}=\frac{\alpha_{s}\left(\alpha_{s}^{2}-s \alpha_{s}-1\right)}{\beta_{s}\left(\beta_{s}^{2}-s \beta_{s}-1\right)} .
$$

- Our arguments will only focus on a neighborhood of a the point $(0: 1: 0)$ at infinity. More precisely we will say that an analytic curve $(x(t), y(t))$ tends to the point at infinity $(0: 1: 0)$ if $y(t) \rightarrow+\infty$ and $\frac{|x(t)|}{|y(t)|} \rightarrow 0$ as $t \rightarrow 0$.

\subsection{Proof in the real case.}

- Fix $t>0$. Let $A, B, C, D, E$ be the following points having all $y$-coordinates equal to $\frac{1}{t}$ :

$-A \in\left(f_{s}=0\right)$ with $x_{A}>0$,

$-B \in \Gamma_{s}:\left(\partial_{x} f_{s}=0\right)$ with $x_{B}>0$,

$-C=\left(0, \frac{1}{t}\right) \in\left(f_{s}=0\right)$,

$-D \in \Gamma_{s}:\left(\partial_{x} f_{s}=0\right)$ with $x_{D}<0$,

- $E \in\left(f_{s}=0\right)$ with $x_{E}<0$.

- Let us fix $s, s^{\prime} \in \mathbb{R}$. By contradiction let us assume that there exists a bilipschitz homeomorphism $\Phi: \mathbb{R}^{2} \rightarrow \mathbb{R}^{2}$ such that $f_{s^{\prime}} \circ \Phi=f_{s}$. Let $K$ be its bilipschitz constant. Let $\tilde{A}, \tilde{B}, \ldots$ be the image by $\Phi$ of $A, B, \ldots$ Let $\gamma$ be the segment $[A B]$ and $\tilde{\gamma}=\Phi(\gamma)$.

- $\Phi$ sends $\left(f_{s}=0\right)$ to $\left(f_{s^{\prime}}=0\right)$ and, as it is a homeomorphism, it should send the component $(x=0)$ of $\left(f_{s}=0\right)$ to the component $(x=0)$ of $\left(f_{s^{\prime}}=0\right)$. Hence $x_{\tilde{C}}=0$.

- $A, B, C, D, E$ and $\gamma$ are all included in the disk of radius $r t$ centered at $C$, where $r$ is a constant that depends only on the fixed value $s$. Hence by the bilipschitz map $\Phi, \tilde{A}, \tilde{B}, \tilde{C}, \tilde{D}, \tilde{E}$ and $\tilde{\gamma}$ are all included in a disk of radius $K r t$ centered at $\tilde{C}$.

- There is an issue: the point $B$ is on the polar curve $\Gamma_{s}$ but $\tilde{B}$ has no reason to be on $\Gamma_{s^{\prime}}$. We will replace $\tilde{B}$ by a point $B^{\prime}$ satisfying this condition. 


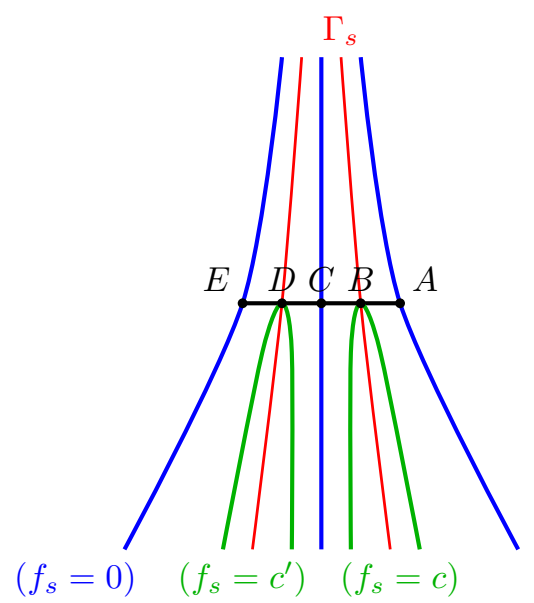

Figure 4. The situation for $f_{s}$.
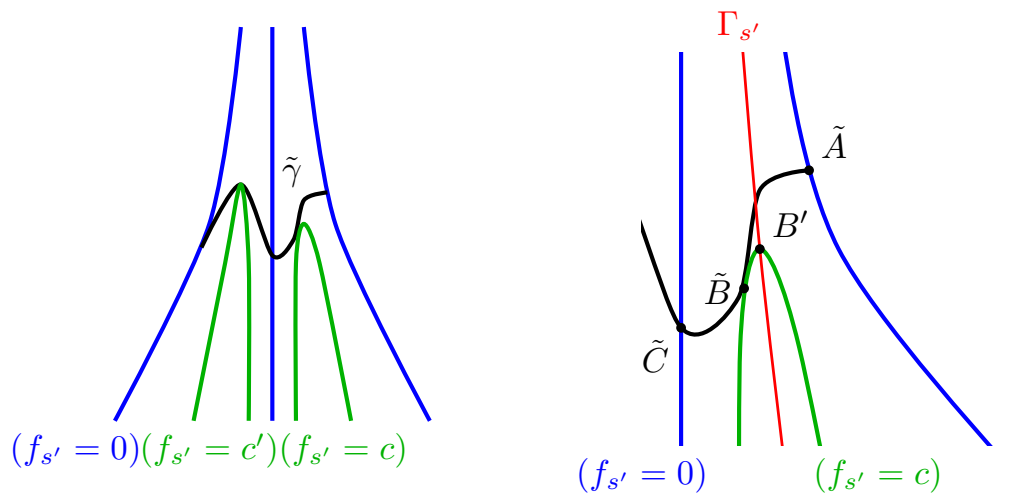

Figure 5. The situation for $f_{s^{\prime}}$.

- Let $c=f_{s}(B)$. Let $\tilde{X}_{c}$ be the part of $\left(f_{s^{\prime}}=c\right)$ in the ball of radius $K r t$ centered at $\tilde{C}$. As $f_{s^{\prime}}(\tilde{B})=f_{s}(B)=c$, then $\tilde{B} \in \tilde{X}_{c}$ and $\tilde{X}_{c}$ is non empty. Moreover $\tilde{X}_{c}$ is contained between two components of $\left(f_{s^{\prime}}=0\right):(x=0)$ and one branch of $\left(x^{2} y^{2}-s^{\prime} x y-1=0\right)$. Moreover $\tilde{X}_{c}$ is strictly below $\tilde{\gamma}$ except at $\tilde{B}$ (because $(f=c)$ is below $\gamma=[A B]$ and intersects it only at $B)$.

- Let $B^{\prime}$ be the point of $\tilde{X}_{c}$ such that $y_{B^{\prime}}$ is maximal among points of $\tilde{X}_{c}$. Then the tangent at $B^{\prime}$ is horizontal, that is to say $\partial_{x} f_{s^{\prime}}\left(B^{\prime}\right)=0$, hence $B^{\prime} \in \Gamma_{s^{\prime}}$. Remember also that $B^{\prime} \in \tilde{X}_{c}$ so that $f_{s^{\prime}}\left(B^{\prime}\right)=c$.

- Partial conclusion: we constructed a point $B^{\prime} \in \Gamma_{s^{\prime}} \cap\left(f_{s^{\prime}}=c\right)$ such that $\left\|B^{\prime}-\tilde{C}\right\| \leqslant$ $K r t$ (with $x_{B^{\prime}}>0$ ).

- We carry on the same proof for the other side. Let $c^{\prime}=f_{s}(D)$, we find a point $D^{\prime} \in \Gamma_{s^{\prime}} \cap\left(f_{s^{\prime}}=c^{\prime}\right)$ such that $\left\|D^{\prime}-\tilde{C}\right\| \leqslant K r t$ (with $\left.x_{D^{\prime}}<0\right)$.

- Now both these points $B^{\prime}$ and $D^{\prime}$ are in the same disk of radius $K r t$ centered at $\tilde{C}$. In particular:

$$
y_{D^{\prime}}-2 K r t \leqslant y_{B^{\prime}} \leqslant y_{D^{\prime}}+2 K r t .
$$


- Let $B^{\prime}=\left(\alpha_{s^{\prime}} t^{\prime}, \frac{1}{t^{\prime}}\right)$ be the coordinates of $B^{\prime}$ on the first branch of $\Gamma_{s^{\prime}}$ and $D^{\prime}=$ $\left(\beta_{s^{\prime}} t^{\prime \prime}, \frac{1}{t^{\prime \prime}}\right)$ be the coordinates of $D^{\prime}$ on the second branch of $\Gamma_{s^{\prime}}$. The former inequalities rewrite:

$$
\frac{1}{t^{\prime \prime}}-2 K r t \leqslant \frac{1}{t^{\prime}} \leqslant \frac{1}{t^{\prime \prime}}+2 K r t .
$$

We consider $t \rightarrow 0$, so that $t^{\prime} \rightarrow 0, t^{\prime \prime} \rightarrow 0$ (a neighborhood of $(0: 1: 0)$ is send to a neighborhood of $(0: 1: 0))$. Hence $t^{\prime \prime}=t^{\prime}+O\left(t t^{\prime} t^{\prime \prime}\right)=t^{\prime}+O\left(t t^{\prime 2}\right)$.

- Now

$$
\begin{gathered}
\frac{f_{s^{\prime}}\left(\alpha_{s^{\prime}} t^{\prime}, \frac{1}{t^{\prime}}\right)}{f_{s^{\prime}}\left(\beta_{s^{\prime}} t^{\prime \prime}, \frac{1}{t^{\prime \prime}}\right)}=\frac{\alpha_{s^{\prime}} t^{\prime}\left(\alpha_{s^{\prime}}^{2}-s^{\prime} \alpha_{s^{\prime}}-1\right)}{\beta_{s^{\prime}} t^{\prime \prime}\left(\beta_{s^{\prime}}^{2}-s^{\prime} \beta_{s^{\prime}}-1\right)} \\
=\frac{\alpha_{s^{\prime}} t^{\prime}\left(\alpha_{s^{\prime}}^{2}-s^{\prime} \alpha_{s^{\prime}}-1\right)}{\beta_{s^{\prime}}\left(t^{\prime}+O\left(t t^{\prime 2}\right)\right)\left(\beta_{s^{\prime}}^{2}-s^{\prime} \beta_{s^{\prime}}-1\right)} \longrightarrow \frac{\alpha_{s^{\prime}}\left(\alpha_{s^{\prime}}^{2}-s^{\prime} \alpha_{s^{\prime}}-1\right)}{\beta_{s^{\prime}}\left(\beta_{s^{\prime}}^{2}-s^{\prime} \beta_{s^{\prime}}-1\right)}
\end{gathered}
$$

as $t^{\prime} \rightarrow 0$.

- On the other hand:

$$
\frac{f_{s^{\prime}}\left(B^{\prime}\right)}{f_{s^{\prime}}\left(D^{\prime}\right)}=\frac{c}{c^{\prime}}=\frac{f_{s^{\prime}}(\tilde{B})}{f_{s^{\prime}}(\tilde{D})}=\frac{f_{s}(B)}{f_{s}(D)}=\frac{\alpha_{s}\left(\alpha_{s}^{2}-s \alpha_{s}-1\right)}{\beta_{s}\left(\beta_{s}^{2}-s \beta_{s}-1\right)} .
$$

Finally:

$$
\frac{\alpha_{s}\left(\alpha_{s}^{2}-s \alpha_{s}-1\right)}{\beta_{s}\left(\beta_{s}^{2}-s \beta_{s}-1\right)}=\frac{\alpha_{s^{\prime}}\left(\alpha_{s^{\prime}}^{2}-s^{\prime} \alpha_{s^{\prime}}-1\right)}{\beta_{s^{\prime}}\left(\beta_{s^{\prime}}^{2}-s^{\prime} \beta_{s^{\prime}}-1\right)} .
$$

- The map $s \mapsto \frac{\alpha_{s}\left(\alpha_{s}^{2}-s \alpha_{s}-1\right)}{\beta_{s}\left(\beta_{s}^{2}-s \beta_{s}-1\right)}=\frac{2\left(s^{2}+3\right) \alpha_{s}+s}{2\left(s^{2}+3\right) \beta_{s}+s}$ is strictly decreasing for $s \in \mathbb{R}$ so that $s=s^{\prime}$.

- Conclusion: if $s, s^{\prime} \in \mathbb{R}$, with $s \neq s^{\prime}$, then there exists no bilipschitz homeomorphism sending $f_{s}$ to $f_{s^{\prime}}$. Since our arguments only care about situation near $(0: 1: 0) f_{s}$ and $f_{s^{\prime}}$ are not right-bilipschitz equivalent at infinity.

3.3. No left-right-equivalence. We now prove that for $s \neq s^{\prime} f_{s}$ and $f_{s^{\prime}}$ are not leftright-equivalent, if we ask the homeomorphism $\Phi$ to be analytic near the point at infinity $(0: 1: 0)$. By contradiction we suppose that there exist bilipschitz homeomorphisms $\Phi$ and $\Psi$ such that $f_{s^{\prime}} \circ \Phi=\Psi \circ f_{s}$ and $\Phi$ is analytic near the point at infinity $(0: 1: 0)$. We continue with the same notation as above, but we cannot conclude as before because we no longer have $\frac{f_{s^{\prime}}\left(B^{\prime}\right)}{f_{s^{\prime}}\left(D^{\prime}\right)}$ equal to $\frac{f_{s}(B)}{f_{s}(D)}$.

- Let $C=\left(0, \frac{1}{t}\right)$ and $\Phi(C)=\tilde{C}=\left(0, \frac{1}{t}\right)(t>0)$. The map $\frac{1}{t} \mapsto \frac{1}{t}$ is a bilipschitz homeomorphism. We will assume $\Phi(0,0)=(0,0)$ so that $\frac{1}{K} \frac{1}{t} \leqslant \frac{1}{t} \leqslant K \frac{1}{t}$ hence $\frac{1}{K} t \leqslant \tilde{t} \leqslant K t$. Define $\chi(t)=\tilde{t}$, for $t>0$, and set $\chi(0)=0$. In the following we will actually only need the relation $\frac{1}{K} t \leqslant \chi(t) \leqslant K t$, but in fact the map $t \mapsto \chi(t)$ is a bilipschitz homeomorphism (with the constant $K^{3}$ ).

- We assumed that the map $\Phi$ is analytic at infinity around $(0: 1: 0)$. It implies that the map $t \mapsto \chi(t)$ is analytic for $t>0: \chi(t)=a_{0} t^{r_{0}}+a_{1} t^{r_{1}}+\cdots$ The map $\chi$ being bilipschitz it implies $r_{0}=1$ so that $\chi(t)=a_{0} t+a_{1} t^{r_{1}}+\cdots$ with $r_{1}>1$.

- Notice that the relation $f_{s^{\prime}} \circ \Phi=\Psi \circ f_{s}$ implies that the map $\Psi$ is also an analytic map.

- Recall that $B=\left(\alpha_{s} t, \frac{1}{t}\right)$ and $f_{s}(B)=c=\alpha_{s}\left(\alpha_{s}^{2}-s \alpha_{s}-1\right) t, D=\left(\beta_{s} t, \frac{1}{t}\right)$ and $f_{s}(D)=c^{\prime}=\beta_{s}\left(\beta_{s}^{2}-s \beta_{s}-1\right) t . \Phi(B)=\tilde{B}$ and $f_{s^{\prime}}(\tilde{B})=\tilde{c}=\Psi(c), \Phi(D)=\tilde{D}$ and $f_{s^{\prime}}(\tilde{D})=\tilde{c}^{\prime}=\Psi\left(c^{\prime}\right)$. We found $B^{\prime}=\left(\alpha_{s^{\prime}} t^{\prime}, \frac{1}{t^{\prime}}\right)$ close to $\tilde{B}$ such that $f_{s^{\prime}}\left(B^{\prime}\right)=$ $f_{s^{\prime}}(\tilde{B})=\tilde{c}$. Hence $\tilde{c}=\alpha_{s^{\prime}}\left(\alpha_{s^{\prime}}^{2}-s^{\prime} \alpha_{s^{\prime}}-1\right) t^{\prime}$. Similarly $D^{\prime}=\left(\beta_{s^{\prime}} t^{\prime \prime}, \frac{1}{t^{\prime \prime}}\right)$ is close to $\tilde{D}$ and $f_{s^{\prime}}\left(D^{\prime}\right)=f_{s^{\prime}}(\tilde{D})=\tilde{c}^{\prime}$. Hence $\tilde{c}^{\prime}=\beta_{s^{\prime}}\left(\beta_{s^{\prime}}^{2}-s^{\prime} \beta_{s^{\prime}}-1\right) t^{\prime \prime}$. 
$B^{\prime}$ is close to $\tilde{B}$ actually means $\left|\frac{1}{t^{\prime}}-\frac{1}{\tilde{t}}\right| \leqslant K r t$, that implies $\left|t^{\prime}-\tilde{t}\right| \leqslant K r t t^{\prime} \tilde{t}$. That implies $t^{\prime}=\chi(t)+O\left(t^{3}\right)$. Similarly $t^{\prime \prime}=\chi(t)+O\left(t^{3}\right)$.

- The map $\Psi$ is defined, for negative values, by $c \mapsto \tilde{c}$ that is to say $\alpha_{s}\left(\alpha_{s}^{2}-s \alpha_{s}-1\right) t \mapsto$ $\alpha_{s^{\prime}}\left(\alpha_{s^{\prime}}^{2}-s^{\prime} \alpha_{s^{\prime}}-1\right) t^{\prime}$. It implies that, for $u<0$, the map $\Psi$ is defined by

$$
\Psi: u \mapsto \alpha_{s^{\prime}}\left(\alpha_{s^{\prime}}^{2}-s^{\prime} \alpha_{s^{\prime}}-1\right) \chi\left(\frac{u}{\alpha_{s}\left(\alpha_{s}^{2}-s \alpha_{s}-1\right)}\right)+O\left(u^{3}\right) .
$$

Hence, as $\chi(t)=a_{0} t+o(t)$ :

$$
\Psi: u \mapsto \frac{\alpha_{s^{\prime}}\left(\alpha_{s^{\prime}}^{2}-s^{\prime} \alpha_{s^{\prime}}-1\right)}{\alpha_{s}\left(\alpha_{s}^{2}-s \alpha_{s}-1\right)} u+o(u) .
$$

Similarly $\Psi(d)=\tilde{d}$ so that for $u>0$ :

$$
\Psi: u \mapsto \frac{\beta_{s^{\prime}}\left(\beta_{s^{\prime}}^{2}-s^{\prime} \beta_{s^{\prime}}-1\right)}{\beta_{s}\left(\beta_{s}^{2}-s \beta_{s}-1\right)} u+o(u) .
$$

- By analycity of $\Psi$, it implies that the coefficients of $u$ are equal, whence

$$
\frac{\alpha_{s}\left(\alpha_{s}^{2}-s \alpha_{s}-1\right)}{\beta_{s}\left(\beta_{s}^{2}-s \beta_{s}-1\right)}=\frac{\alpha_{s^{\prime}}\left(\alpha_{s^{\prime}}^{2}-s^{\prime} \alpha_{s^{\prime}}-1\right)}{\beta_{s^{\prime}}\left(\beta_{s^{\prime}}^{2}-s^{\prime} \beta_{s^{\prime}}-1\right)},
$$

which is impossible for $s \neq s^{\prime}$ as we have seen before in section 3.2.

3.4. No left-right-equivalence (again). It is not clear whether $f_{s}$ and $f_{s^{\prime}}\left(s \neq s^{\prime}\right)$ are or not left-right bilipschitz equivalent when no restriction is made on $\Phi$. However we can complicate our example in order to exclude left-right equivalence.

Lemma 4. Let

$$
f_{s}(x, y)=x\left(x^{4} y^{4}-3 s x^{2} y^{2}+1\right)
$$

be a family of polynomials in $\mathbb{R}[x, y]$. Then for $s, s^{\prime}>1$, with $s \neq s^{\prime}$, the polynomials $f_{s}$ and $f_{s^{\prime}}$ are not left-right bilipschitz equivalent.

Proof. The proof is similar to the proof of section 3.3.

- The equation $5 z^{4}-9 s z^{2}+1=0$ has 4 real solutions $-\alpha_{s}<-\beta_{s}<\beta_{s}<\alpha_{s}$ corresponding to 4 branches of the polar curve $\left(\partial_{x} f_{s}=0\right)$.

- We use the same method as before in section 3.3 with $B=\left(-\alpha_{s} t, \frac{1}{t}\right), f_{s}(B)=$ $-\alpha_{s}\left(\alpha_{s}^{4}-3 s \alpha_{s}^{2}+1\right) t=c_{s} t>0$ and $D=\left(\beta_{s} t, \frac{1}{t}\right), f_{s}(D)=\beta_{s}\left(\beta_{s}^{4}-3 s \beta_{s}^{2}+1\right) t=$ $d_{s} t>0$ (with $t>0$ ).

- This times for $u>0$ we have two formulas for $\Psi$ :

$$
\Psi(u)=c_{s^{\prime}} \chi\left(\frac{u}{c_{s}}\right)+O\left(u^{3}\right),
$$

and

$$
\Psi(u)=d_{s^{\prime}} \chi\left(\frac{u}{d_{s}}\right)+O\left(u^{3}\right) .
$$

- It implies that the bilipschitz map $\chi$ verifies

$$
\chi\left(\frac{c_{s}}{d_{s}} v\right)=\frac{c_{s^{\prime}}}{d_{s^{\prime}}} \chi(v)+O\left(v^{3}\right)
$$

for all $v>0$ near 0 .

- Then by lemma 5 below, it implies $p=\frac{c_{s}}{d_{s}}>1$ is equal to $q=\frac{c_{s^{\prime}}}{d_{s^{\prime}}}>1$ which is impossible if $s \neq s^{\prime}$. 
Lemma 5. Let $\chi: \mathbb{R} \rightarrow \mathbb{R}$ be a bilipschitz map such that

$$
\chi(p v)=q \chi(v)+O\left(v^{3}\right)
$$

for some constant $p, q>1$ and all $v$ near 0 . Then $p=q$.

Proof. We have $\chi(v)=q \chi(v / p)+v^{3} \eta(v)$, where $\eta(v)$ is a bounded function for $v$ near 0 . By induction it yields $\chi(v)=q^{n} \chi\left(v / p^{n}\right)+v^{3} \sum_{k=0}^{n-1} \eta\left(v / p^{k}\right)\left(q / p^{3}\right)^{k}$. Hence, except for the special case $p^{3}=q$ that would be treated in a similar way, we have:

$$
\left|\chi(v)-q^{n} \chi\left(\frac{v}{p^{n}}\right)\right| \leqslant C v^{3} \frac{1-\left(q / p^{3}\right)^{n}}{1-q / p^{3}} .
$$

Let $K>0$ be a bilipschitz constant for $\chi$. As $\chi(0)=0$ we have $K^{-1}<\frac{|\chi(v)|}{|v|}<K$ for all $v \neq 0$. In particular $K^{-1}<p^{n} \frac{\left|\chi\left(v / p^{n}\right)\right|}{|v|}<K$.

Case $p>q$. Then we have $q^{n} \chi\left(v / p^{n}\right) \rightarrow 0$ as $n \rightarrow+\infty$. At the limit, when $n \rightarrow+\infty$, inequality (1) gives $|\chi(v)| \leqslant C^{\prime} v^{3}$, which contradicts that $\chi$ is bilipschitz.

Case $p<q$. Inequality (1) gives

$$
\left|\frac{p^{n}}{q^{n}} \chi(v)-p^{n} \chi\left(\frac{v}{p^{n}}\right)\right| \leqslant C^{\prime} v^{3}\left(\frac{p^{n}}{q^{n}}-\frac{1}{p^{2 n}}\right)
$$

Fix $v \neq 0$. As $n \rightarrow+\infty$, the term $p^{n} \chi\left(\frac{v}{p^{n}}\right)$ does not tend towards 0 , it contradicts that all the other terms $\frac{p^{n}}{q^{n}} \chi(v), \frac{p^{n}}{q^{n}}$ and $\frac{1}{p^{2 n}}$ tends towards 0 .

Conclusion: $p=q$.

We completed the proof of theorem 1 in the real setting.

\section{Proof in the COMPlex CASE}

The proof in the complex case at infinity is an adaptation of the local proof of Henry and Parusiński [5].

\subsection{Notations.}

- Let $g: \mathbb{C}^{2} \rightarrow \mathbb{C}$ be a polynomial map and $p=(x, y)$ be a point near the point at infinity $(0: 1: 0)$, that is to say $|y| \gg 1$ and $|x| \ll|y|$.

- Fix $p_{0}$, let $c=g\left(p_{0}\right)$. Denote $B\left(p_{0}, \rho\right)$ the open ball centered at $p_{0}$ of radius $\rho$ and $X\left(p_{0}, \rho\right)=(g=c) \cap B\left(p_{0}, \rho\right)$.

- Fix $K>0$ and denote $\operatorname{dist}_{p_{0}, \rho, K}(p, q)$ the inner distance of $p$ and $q$ supposed to be in the same connected component of $X\left(p_{0}, K \rho\right)$.

- Let

$$
\phi\left(p_{0}, K, \rho\right)=\sup \frac{\operatorname{dist}_{p_{0}, \rho, K}(p, q)}{\|p-q\|}
$$

be the ratio between the inner and outer distances.

- Denote

- Finally let

$$
\psi\left(p_{0}, K, \rho\right)=\sup _{\rho^{\prime} \leqslant \rho} \phi\left(p_{0}, K, \rho^{\prime}\right)
$$

$$
Y(\rho, K, A)=\{p \mid \psi(p, K, \rho) \geqslant A\} .
$$

be the set of points $p$ where the curvature of the curve $(g=c)$ is large.

- Let $\Phi: \mathbb{C}^{2} \rightarrow \mathbb{C}^{2}$ be a bilipschitz homeomorphism at infinity such that $\tilde{g} \circ \Phi=g$. Let $L$ be a bilipschitz constant of $\Phi$. 
- Once $\Phi$ is fixed, we add a tilde to denote an object in then target space, for instance

$$
\tilde{Y}(\rho, K, A)=\{\tilde{p} \mid \psi(\tilde{p}, K, \rho) \geqslant A\}
$$

is the set of points $\tilde{p}$ in the target space where the curvature of the curve $(\tilde{g}=\tilde{c})$ is large.

We have the following lemma saying that points with large curvature are sent to points of large curvature by a bilipschitz map:

Lemma 6 ([5], Lemma 2.1). For $K \geqslant L^{2}$ :

$$
\tilde{Y}\left(L^{-1} \rho, K, A L^{2}\right) \subset \Phi(Y(\rho, K, A)) \subset \tilde{Y}\left(L \rho, K, A L^{-2}\right) .
$$

And a variant:

Lemma 7 ([5], Lemma 2.2). Let $\delta>0$ and

$$
Y(\delta, K, M, A)=\left\{p \mid \psi\left(p, M\|p\|^{-1+\delta}, K\right) \geqslant A\right\} .
$$

If $K \geqslant L^{2}$ then:

$$
\tilde{Y}\left(\delta, K, M L^{-\delta}, A L^{2}\right) \subset \Phi(Y(\delta, K, M, A)) \subset \tilde{Y}\left(\delta, K, M L^{+\delta}, A L^{-2}\right) .
$$

Remarks:

- There are two distinct uses of the norm:

- $\|p-q\|:$ distance between two "near" points: a "small" number.

- $\|p\|$ : distance to the origin: a "large" number. We will use it for $\frac{1}{\|p\|}$ in order to get a "small" number.

- If we denote $\tilde{p}=\Phi(p)$, then the bilipschitz property implies: $L^{-1}\|p\| \leqslant\|\tilde{p}\| \leqslant L\|p\|$ for some bilipschitz constant $L$, hence also:

$$
L^{-1}\|p\|^{-1} \leqslant\|\tilde{p}\|^{-1} \leqslant L\|p\|^{-1} .
$$

- Notice that in our definition of $Y(\delta, K, M, A)$ of lemma 7 there is a term in $\|p\|^{-1+\delta}$ while in [5] the term is $\|p\|^{1+\delta}$. After this modification, the proof is the same as in [5].

- We will restrict ourselves to a neighborhood of the point at infinity $(0: 1: 0)$, in particular we may suppose $|y| \gg|x|$ so that morally $\|p\|=\|(x, y)\| \simeq|y|$ (this is an equality in the case $\left.\|\cdot\|=\|\cdot\|_{\infty}\right)$.

Fix $s \in \mathbb{C}$ and denote $f_{s}(x, y)=x\left(x^{2} y^{2}-s x y-1\right)$. Let us denote $U=\left\{(x, y)|| \partial_{x} f_{s} \mid<\right.$ $\left.\left|\partial_{y} f_{s}\right|\right\}$.

Lemma 8 (compare to [5], Lemma 3.2). Let $(x(t), y(t)) \in U$ with $y(t)=\frac{1}{t}$. Then for $s^{2}+3 \neq 0$ :

$$
x(t)=\gamma t+O\left(t^{3}\right) \quad \text { and } \quad f_{s}(x(t), y(t))=\gamma\left(\gamma^{2}-s \gamma-1\right) t+O\left(t^{3}\right),
$$

with

$$
\gamma=\alpha_{s} \text { or } \gamma=\beta_{s} \text { a solution of } 3 z^{2}-2 s z-1=0 .
$$

In this section we now suppose $s^{2}+3 \neq 0$.

Proof. Let $u=x y$. On $U$ the inequality $\left|\partial_{x} f_{s}\right|<\left|\partial_{y} f_{s}\right|$ yields $\left|3 u^{2}-2 s u-1\right|<|x|^{2}|2 u-s|$. In a neighborhood of the point at infinity $(0: 1: 0)$ we first prove that $|x(t)|$ is bounded as $t \rightarrow+\infty$. If this is not the case, then write $x(t)=a_{0} t^{r_{0}}+a_{1} t^{r_{1}}+\cdots$ with $r_{i} \in$ $\mathbb{Q}, r_{i}<r_{i+1}$ and here $r_{0}<0$. As $y(t)=1 / t$, then $u(t) \sim a_{0} t^{r_{0}-1} \rightarrow+\infty$. Then $\left|3 u^{2}-2 s u-1\right|<|x|^{2}|2 u-s|$ implies $r_{0} \leqslant-1$ in contradiction with $\frac{x(t)}{y(t)} \rightarrow 0$. Now, 
as $|x(t)|$ is bounded, inequality $\left|3 u^{2}-2 s u-1\right|<|x|^{2}|2 u-s|$ implies that $|u(t)|$ is also bounded. Write again $x(t)=a_{0} t^{r_{0}}+a_{1} t^{r_{1}}+\cdots$ and using that $u(t)$ is bounded gives $r_{0} \geqslant 1: x(t)=a_{0} t+a_{1} t^{r_{1}}+\cdots$ and $u(t)=a_{0}+a_{1} t^{r_{1}-1}+\cdots\left(a_{0} \in \mathbb{C}\right)$. We plug $u(t)$ in the inequality $\left|3 u^{2}-2 s u-1\right|<|x|^{2}|2 u-s|$ :

$$
\left|3\left(a_{0}+a_{1} t^{r_{1}-1}+\cdots\right)^{2}-2 s\left(a_{0}+a_{1} t^{r_{1}-1}+\cdots\right)-1\right|=O\left(t^{2}\right) .
$$

It implies:

$$
3 a_{0}^{2}-2 s a_{0}-1=0
$$

and

$$
6 a_{0} a_{1} t^{r_{1}-1}-2 s a_{1} t^{r_{1}-1}=O\left(t^{2}\right) .
$$

We may suppose $a_{1} \neq 0$ and we now prove $r_{1} \geqslant 3$. Otherwise $6 a_{0}=2 s$, that is to say $s=3 a_{0}$, but $a_{0}$ is a solution of $3 z^{2}-2 s z-1=0$. This is only possible if $s^{2}+3=0$. So that $x(t)=\gamma t+O\left(t^{3}\right)$ as required, where $\gamma$ is a solution of $3 z^{2}-2 s z-1=0$. Then $f_{s}(x(t), y(t))=\gamma\left(\gamma^{2}-s \gamma-1\right) t+O\left(t^{3}\right)$.

Lemma 9 (compare to [5], Lemma 3.3). Let $0<\delta<1$ and $C>0$. On the set:

$$
\left\{p=(x, y)\left|\exists p_{0}=\left(x_{0}, y_{0}\right) \in U, f_{s}(p)=f_{s}\left(p_{0}\right),\right| y-\left.y_{0}|\leqslant C| y_{0}\right|^{-1+\delta}\right\},
$$

if we denote $y(t)=\frac{1}{t}$, then

$$
x(t)=O(t)
$$

and

$$
f_{s}(x(t), y(t))=\gamma\left(\gamma^{2}-s \gamma-1\right) t+O\left(t^{2-\delta}\right) .
$$

Proof. We denote $y(t)=\frac{1}{t}$ and $y\left(t_{0}\right)=\frac{1}{t_{0}}$. As $\left|y-y_{0}\right| \leqslant C\left|y_{0}\right|^{-1+\delta}$, we have $\left|\frac{1}{t}-\frac{1}{t_{0}}\right| \leqslant$ $C\left|t_{0}\right|^{1-\delta}$ hence $\left|t_{0} / t-1\right| \leqslant C\left|t_{0}\right|^{2-\delta}$ hence $t_{0} / t \rightarrow 1$, i.e $t \sim t_{0}$. Then $\left|t_{0} / t-1\right| \leqslant C^{\prime}|t|^{2-\delta}$ so that $t_{0}=t+O\left(t^{2-\delta}\right)$.

Now by hypothesis and by lemma 8 ,

$$
f_{s}(x(t), y(t))=f_{s}\left(x\left(t_{0}\right), y\left(t_{0}\right)\right)=\gamma\left(\gamma^{2}-s \gamma-1\right) t_{0}+O\left(t_{0}^{3}\right)=\gamma\left(\gamma^{2}-s \gamma-1\right) t+O\left(t^{2-\delta}\right) .
$$

So that

$$
f_{s}(x(t), y(t))=x(t)\left(x(t)^{2} y(t)^{2}-s x(t) y(t)-1\right)=\gamma\left(\gamma^{2}-s \gamma-1\right) t+O\left(t^{2-\delta}\right) .
$$

We start over the computations of lemma 8. Set $x(t)=a_{0} t^{r_{0}}+a_{1} t^{r_{1}}+\cdots$ and $y(t)=1 / t$. Then

$$
\frac{x(t)}{t}\left(\frac{x(t)^{2}}{t^{2}}-s \frac{x(t)}{t}-1\right)=\gamma\left(\gamma^{2}-s \gamma-1\right)+O\left(t^{1-\delta}\right)
$$

We cannot have $r_{0}>1$ since we would have $\frac{x(t)}{t} \rightarrow 0$ (as $t \rightarrow 0$ ) and the left-hand side of equation (4) would also tends to 0 . We cannot either have $r_{0}<1$, since we would have $\left|\frac{x(t)}{t}\right| \rightarrow+\infty$ and the left-hand side of equation (4) would also tends to infinity. Then $r_{0}=1$ and $a_{0}\left(a_{0}^{2}-s a_{0}-1\right)=\gamma\left(\gamma^{2}-s \gamma-1\right)$, so that $x(t)=O(t)$.

Lemma 10 (compare to [5], Corollary 3.4). Let $Y=Y(\delta, K, M, A)=\left\{p \mid \psi\left(p, M\|p\|^{-1+\delta}, K\right) \geqslant\right.$ $A\}$ where $0<\delta<1, M>0$ and $A, K$ are sufficiently large constants. Then the formulas (2) and (3) holds for $(x(t), y(t)) \in Y$ with $y(t)=\frac{1}{t}$. 
Proof. The proof is the same as in [5]: for $p_{0}=\left(x_{0}, y_{0}\right) \in Y$ there exists $p=(x, y) \in U$ such that

$$
\left\|p-p_{0}\right\| \leqslant K M\left\|p_{0}\right\|^{-1+\delta} .
$$

As $\frac{1}{2}\left|y_{0}\right| \leqslant\left\|p_{0}\right\| \leqslant 2\left|y_{0}\right|$ (since $x_{0} \leqslant y_{0}$ ), it implies $\left|y-y_{0}\right| \leqslant C\left|y_{0}\right|^{-1+\delta}$ and lemma 9 applies.

Lemma 11 (compare to [5], Proposition 3.5). Let $Y=Y(\delta, K, M, A)$, where $0<\delta<1$, $M>0$ and $A, K$ are sufficiently large constants. Suppose that $p_{1}$ and $p_{2}$ are in $Y$ and there exists a $0<\delta_{1}<1$ such that $\left\|p_{1}-p_{2}\right\| \leqslant\left\|p_{1}\right\|^{-1+\delta_{1}}$. Then for $\max \left\{\delta, \delta_{1}\right\}<\delta_{2}<1$ and in a sufficiently small neighborhood of the point at infinity $(0: 1: 0)$ :

with

$$
\left|\frac{f_{s}\left(p_{1}\right)}{f_{s}\left(p_{2}\right)}-a\right| \leqslant\left\|p_{1}\right\|^{-1+\delta_{2}}
$$

$$
a \in\left\{1, \frac{\alpha_{s}\left(\alpha_{s}^{2}-s \alpha_{s}-1\right)}{\beta_{s}\left(\beta_{s}^{2}-s \beta_{s}-1\right)}, \frac{\beta_{s}\left(\beta_{s}^{2}-s \beta_{s}-1\right)}{\alpha_{s}\left(\alpha_{s}^{2}-s \alpha_{s}-1\right)}\right\} .
$$

Proof. Let $p_{1}=\left(x_{1}(t), y_{1}(t)\right)$ and $p_{2}=\left(x_{2}\left(t^{\prime}\right), y_{2}\left(t^{\prime}\right)\right)$ be two points in $Y$. Then by lemma 10

$$
\begin{gathered}
f_{s}\left(x_{1}(t), y_{1}(t)\right)=\gamma\left(\gamma^{2}-s \gamma-1\right) t+O\left(t^{2-\delta}\right), \\
f_{s}\left(x_{2}\left(t^{\prime}\right), y_{2}\left(t^{\prime}\right)\right)=\gamma^{\prime}\left(\gamma^{\prime 2}-s \gamma^{\prime}-1\right) t^{\prime}+O\left(t^{\prime 2-\delta}\right),
\end{gathered}
$$

where $\gamma$ and $\gamma^{\prime}$ are in $\left\{\alpha_{s}, \beta_{s}\right\}$.

Now as $\left\|p_{1}-p_{2}\right\| \leqslant\left\|p_{1}\right\|^{-1+\delta_{1}}$ it implies $\left|y_{1}-y_{2}\right| \leqslant 2\left|y_{1}\right|^{-1+\delta_{1}}$, as in the proof of lemma 9 we get $t^{\prime}=t+O\left(t^{2-\delta_{1}}\right)$. Whence

$$
f_{s}\left(x_{2}\left(t^{\prime}\right), y_{2}\left(t^{\prime}\right)\right)=\gamma^{\prime}\left(\gamma^{\prime 2}-s \gamma^{\prime}-1\right) t+O\left(t^{2-\delta_{1}}\right)+O\left(t^{2-\delta}\right) .
$$

Then

$$
\frac{f_{s}\left(p_{1}\right)}{f_{s}\left(p_{2}\right)}=\frac{\gamma\left(\gamma^{2}-s \gamma-1\right)}{\gamma^{\prime}\left(\gamma^{\prime 2}-s \gamma^{\prime}-1\right)}+O\left(t^{1-\delta_{1}}\right)+O\left(t^{1-\delta}\right) .
$$

Then for $\delta_{2}>\max \left\{\delta, \delta_{1}\right\}$ with $\delta_{2}<1$ and in neighborhood of the point at infinity $(0: 1: 0)$ we get:

where $a=\gamma / \gamma^{\prime}$.

$$
\left|\frac{f_{s}\left(p_{1}\right)}{f_{s}\left(p_{2}\right)}-a\right| \leqslant \frac{1}{2}|t|^{1-\delta_{2}} \leqslant\left\|p_{1}\right\|^{-1+\delta_{2}}
$$

Lemma 12 (compare to [5], Lemma 3.6). Let $K$ and $A$ sufficiently large and $0<\delta<1$. Fix $s$ with $s^{2}+3 \neq 0$. Then $Y=Y(\delta, K, M, A)$ is nonempty and contains the polar curve $\Gamma_{s}$. Moreover all the limits of $f_{s}\left(p_{1}\right) / f_{s}\left(p_{2}\right)$ given in lemma 11 can be obtained by taking $p_{1}$ and $p_{2}$ along the branches of $\Gamma_{s}$ associated to the point at infinity $(0: 1: 0)$.

Proof. Fix $\delta$ and $K$. Let $\pi_{c}:\left(f_{s}=c\right) \rightarrow \mathbb{C}$ be the projection $(x, y) \mapsto y$. It is a triple covering branched at the points $\Gamma_{s} \cap\left(f_{s}=c\right)$. These points are of coordinates

$$
\left(\alpha_{s} t, \frac{1}{t}\right) \quad \text { and } \quad\left(\beta_{s} t^{\prime}, \frac{1}{t^{\prime}}\right) \quad \text { with } \quad f_{s}\left(\alpha_{s} t, \frac{1}{t}\right)=f_{s}\left(\beta_{s} t^{\prime}, \frac{1}{t^{\prime}}\right)=c .
$$

As $f_{s}\left(\alpha_{s} t, \frac{1}{t}\right)=\alpha_{s} t\left(\alpha_{s}^{2}-s \alpha_{s}-1\right)$ it implies

$$
t=\frac{c}{\alpha_{s}\left(\alpha_{s}^{2}-s \alpha_{s}-1\right)} \quad \text { and similarly } \quad t^{\prime}=\frac{c}{\beta_{s}\left(\beta_{s}^{2}-s \beta_{s}-1\right)} .
$$

For $s^{2}+3 \neq 0, \alpha_{s} \neq \beta_{s}$ and it also implies $t \neq t^{\prime}$ hence $\left|y(t)-y\left(t^{\prime}\right)\right|$ is of order $y(t)$, that is to say two points of ramifications are far enough. Let $p_{0}=\left(x_{0}, y_{0}\right)$ be a point of ramification 
of $\pi_{c}$. Let $\mathcal{V}=\left\{y|| y-y_{0}|\leqslant \epsilon| y_{0} \mid\right\}$, with $\epsilon$ sufficiently small such that no other ramification point projects in $\mathcal{V}$. For a sufficiently large $p_{0}$ (i.e. small $c$ ), $X\left(p_{0}, K M\left\|p_{0}\right\|^{-1+\delta}\right) \subset \pi_{c}^{-1}(\mathcal{V})$. Now let $p=(x, y)$ such that:

$$
\left|y-y_{0}\right| \leqslant\left|y_{0}\right|^{-1+\delta}
$$

then by lemma $9, x=O\left(\frac{1}{y}\right)$ whence

$$
\left\|p-p_{0}\right\| \leqslant 2\left\|p_{0}\right\|^{-1+\delta} .
$$

Let $\mathcal{V}_{\delta}=\left\{y|| y-\left.y_{0}|\leqslant \epsilon| y_{0}\right|^{-1+\delta}\right\}$, by the above inequality we get $\pi_{c}^{-1}\left(\mathcal{V}_{\delta}\right) \subset X\left(p_{0}, K M\left\|p_{0}\right\|^{-1+\delta}\right)$. We restrict the triple branched covering $\pi_{c}$ to a map $\tilde{\pi}_{c}$ from $\pi_{c}^{-1}\left(\mathcal{V}_{\delta}\right)$ composed by only two components of the triple cover. Let $y \in \mathcal{V}_{\delta}$ such that $\left|y-y_{0}\right|=\frac{1}{2}\left|y_{0}\right|^{-1+\delta}$. Let $p_{1}=\left(x_{1}, y\right), p_{2}=\left(x_{2}, y\right)$ be the two points of $\tilde{\pi}_{c}^{-1}(y)$. These two points are in $\tilde{\pi}_{c}^{-1}\left(\mathcal{V}_{\delta}\right)$ which is a connected set. Any curve $\gamma$ in $\tilde{\pi}_{c}^{-1}\left(\mathcal{V}_{\delta}\right)$ from $p_{1}$ to $p_{2}$ passes through $p_{0}$, hence the projection of $\gamma$ by $\tilde{\pi}_{c}$ passes through $y_{0}$. Hence the inner distance (in $\left(f_{s}=c\right)$ ) of $p_{1}$ and $p_{2}$ is greater or equal than $2\left|y-y_{0}\right|$, it yields:

$$
\operatorname{dist}_{p_{0}, M\left\|p_{0}\right\|^{-1+\delta}, K}\left(p_{1}, p_{2}\right) \geqslant 2\left|y-y_{0}\right|=\left|y_{0}\right|^{-1+\delta}=|t|^{1-\delta},
$$

where we denote $y_{0}=\frac{1}{t}$. By lemma 9 we have $x_{1}=O(t)$ and $x_{2}=O(t)$, so that

$$
\left\|p_{1}-p_{2}\right\| \leqslant C|t| \text {. }
$$

Then

$$
\frac{\operatorname{dist}_{p_{0}, M\left\|p_{0}\right\|^{-1+\delta}, K}\left(p_{1}, p_{2}\right)}{\left\|p_{1}-p_{2}\right\|} \geqslant \frac{1}{C|t|^{\delta}} \underset{t \rightarrow 0}{\longrightarrow}+\infty .
$$

Then $\psi\left(p_{0}, M\left\|p_{0}\right\|^{-1+\delta}, K\right) \rightarrow+\infty$, as $p_{0}$ tends to the point at infinity $(0: 1: 0)$. It means that the branch of $\Gamma_{s}$ near this point at infinity is included in $Y(\delta, K, M, A)$.

Finally we have already proved in subsection 3.1 that the list of values $f_{s}\left(p_{1}\right) / f_{s}\left(p_{2}\right)$ on $\Gamma_{s}$ is the required one.

We conclude by the proof of the theorem in the complex case.

Proof of theorem 1'. Fix $s$. By lemma 7 the set $Y$ for $f_{s}$ is sent into a set $\tilde{Y}$ for $f_{s^{\prime}}$. The polar curve $\Gamma_{s}$ is included in $Y$ (lemma 12) and on this polar curve $f_{s}\left(p_{1}\right) / f_{s}\left(p_{2}\right)$ tends to a $\frac{\alpha_{s}\left(\alpha_{s}^{2}-s \alpha_{s}-1\right)}{\beta_{s}\left(\beta_{s}^{2}-s \beta_{s}-1\right)}$ for instance (lemma 11). On the one hand $f_{s^{\prime}}\left(\tilde{p}_{1}\right) / f_{s^{\prime}}\left(\tilde{p}_{2}\right)$ tends to the same value, because the bilipschitz homeomorphism $\Phi$ sends the levels of $f_{s}$ to the levels of $f_{s^{\prime}}$. On the other hand $\tilde{p}_{1}, \tilde{p}_{2}$ are in $\tilde{Y}$ so that $f_{s^{\prime}}\left(\tilde{p}_{1}\right) / f_{s^{\prime}}\left(\tilde{p}_{2}\right)$ is in

$$
\left\{1, \frac{\alpha_{s^{\prime}}\left(\alpha_{s^{\prime}}^{2}-s^{\prime} \alpha_{s^{\prime}}-1\right)}{\beta_{s^{\prime}}\left(\beta_{s^{\prime}}^{2}-s^{\prime} \beta_{s^{\prime}}-1\right)}, \frac{\beta_{s^{\prime}}\left(\beta_{s^{\prime}}^{2}-s^{\prime} \beta_{s^{\prime}}-1\right)}{\alpha_{s^{\prime}}\left(\alpha_{s^{\prime}}^{2}-s^{\prime} \alpha_{s^{\prime}}-1\right)}\right\} \text {. }
$$

This is only possible for a finite set of values $s^{\prime}$.

\section{Topological EQUivalence}

To complete the complex part of theorem 1 we prove the topological equivalence of any two polynomials.

Lemma 13. Consider the following family of polynomials in $\mathbb{C}[x, y]$ :

$$
f_{s}(x, y)=x\left(x^{2} y^{2}-s x y-1\right)
$$

with $s^{2}+4 \neq 0$. For any $s$ and $s^{\prime}$ the polynomials $f_{s}$ and $f_{s^{\prime}}$ are topologically equivalent. 
This family is similar to examples in [1] of polynomials that are topologically equivalent but not algebraically equivalent. Recall that two polynomials $f, g: \mathbb{K}^{n} \rightarrow \mathbb{K}$ are topologically equivalent if there exist a homeomorphism $\Phi: \mathbb{K}^{n} \rightarrow \mathbb{K}^{n}$ and a homeomorphism $\Psi: \mathbb{K} \rightarrow \mathbb{K}$ such that $g \circ \Phi=\Psi \circ f$. We will use the following result that is global version of Lê-Ramanujam $\mu$-constant theorem. See [2] for the two variables case and [3] for any number of variables.

Theorem 14. Let $\left\{f_{s}\right\}_{s \in[0,1]}$ be a continuous family of complex polynomials with isolated singularities (in the affine space and at infinity), with $n \neq 3$ variables. Suppose that the following integers are constant w.r.t. the value of $s$ :

- $\operatorname{deg} f_{s}$, the degree,

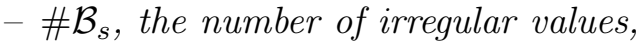

$-\chi\left(f_{s}=c_{g e n}\right)$, the Euler characteristic of a generic fiber.

Then $f_{0}$ and $f_{1}$ are topologically equivalent.

Proof of lemma 13.

- Degree. It is clear that the degree of the $f_{s}$ is independent of $s$.

- Affine singularities. We search for points $(x, y)$ where both derivatives vanish. $\partial_{x} f_{s}(x, y)=3 x^{2} y^{2}-2 s x y-1$ and $\partial_{y} f_{s}(x, y)=x^{2}(2 x y-s)$. If $x=0$ then $\partial_{x} f_{s}(x, y) \neq 0$. So that $\partial_{y} f_{s}(x, y)=0$ implies $2 x y-s=0$. We plug $x y=s / 2$ in $\partial_{x} f_{s}(x, y)=0$ and get $s^{2}+4=0$. Notice that $s^{2}+4=0$ gives also the values where $f_{s}$ is not a reduced polynomial. Conclusion: for $s^{2}+4 \neq 0$, the polynomials $f_{s}$ has no affine singularities (nor affine critical values), so that its global affine Milnor number is $\mu_{s}=0$.

- Singularities at infinity. The two points at infinity for this family are $P_{1}=(0$ : $1: 0)$ and $P_{2}=(1: 0: 0)$. Let $F_{s}(x, y, z)=x\left(x^{2} y^{2}-s x y z^{2}-z^{4}\right)-c z^{5}$ be the homogenization of $f_{s}(x, y)-c$.

- Milnor number at $P_{1}$. We localize $F_{s}$ at $P_{1}=(0: 1: 0)$ to get $g_{s}(x, z)=$ $F_{s}(x, 1, z)=x\left(x^{2}-s x z^{2}-z^{4}\right)-c z^{5}$. We compute the local Milnor of $g_{s}$ at $(0,0)$. For instance we may use the Newton polygon of $g_{s}$ and Kouchnirenko formula. We get, for any $s$ (with $s^{2}+4 \neq 0$ ) and depending on $c$ :

$$
\mu\left(g_{s}\right)=8 \text { if } c \neq 0 \text { and } \mu\left(g_{s}\right)=10 \text { if } c=0 .
$$

Hence the value 0 is an irregular value at infinity and the jump of Milnor number is $\lambda_{P_{1}}=10-8=2$.

- Milnor number at $P_{2}$. At $P_{2}=(1: 0: 0)$ we get $h_{s}(y, z)=F_{s}(1, y, z)=$ $y^{2}-s y z^{2}-z^{4}-c z^{5}$. The local Milnor number of $h_{s}$ at $(0,0)$ is independent of $s$ and $c$ :

$$
\mu\left(h_{s}\right)=3 .
$$

So that there is no irregular values at infinity for this point and $\lambda_{P_{2}}=0$.

- Then the Milnor number at infinity is $\lambda_{s}=\lambda_{P_{1}}+\lambda_{P_{2}}=2$ and the only irregular value at infinity is 0 .

- Conclusion. For all $s$ the only irregular value is $0: \mathcal{B}_{s}=\{0\}$, the Euler characteristic of a generic fiber given by $\chi_{s}=1-\mu_{s}-\lambda_{s}=-1$ is also constant. Then by theorem 14 any $f_{s}$ and $f_{s^{\prime}}$ are topologically equivalent. 


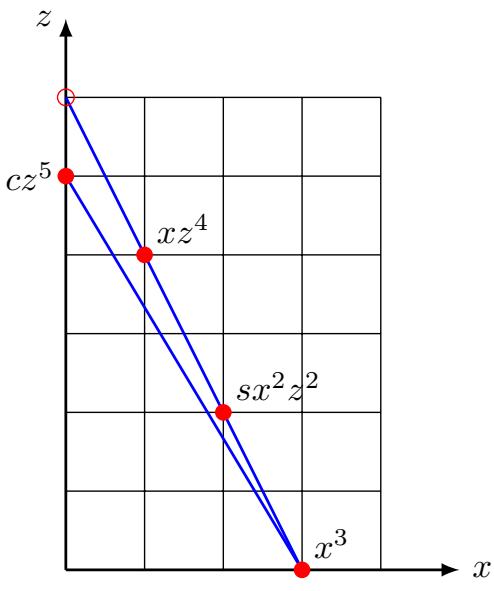

Newton polygon of $g_{s}(x, z)$

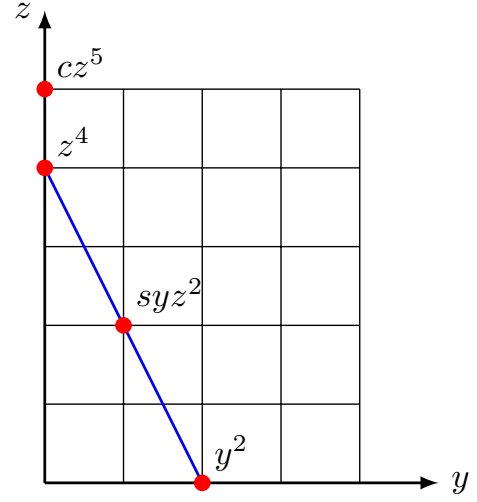

Newton polygon of $h_{s}(y, z)$

FiguRE 6. Computation of Milnor number at infinity.

\section{REFERENCES}

[1] Arnaud Bodin. Classification of polynomials from $\mathbb{C}^{2}$ to $\mathbb{C}$ with one critical value. Math. Z., 242(2):303322, 2002.

[2] Arnaud Bodin. Invariance of Milnor numbers and topology of complex polynomials. Comment. Math. Helv., 78(1):134-152, 2003.

[3] Arnaud Bodin and Mihai Tibăr. Topological equivalence of complex polynomials. Adv. Math., 199(1):136-150, 2006.

[4] Takuo Fukuda. Types topologiques des polynômes. Inst. Hautes Études Sci. Publ. Math., (46):87-106, 1976.

[5] Jean-Pierre Henry and Adam Parusiński. Existence of moduli for bi-Lipschitz equivalence of analytic functions. Compositio Math., 136(2):217-235, 2003.

E-mail address: arnaud.bodin@univ-lille.fr

Université de Lille, CNRS, Laboratoire Paul Painlevé, 59000 Lille, France 